\title{
Thermophysical properties of Ni-containing single-phase concentrated solid solution alloys
}

\author{
K. Jin ${ }^{1}$, S. Mu ${ }^{1}$, K. An ${ }^{2}$, W. D. Porter $^{1}$, G. D. Samolyuk ${ }^{1}$, G. M. Stocks ${ }^{1}$, and H. Bei ${ }^{1 *}$ \\ 1 Materials Science \& Technology Division, Oak Ridge National Laboratory, Oak Ridge, TN \\ 37831, USA \\ 2 Chemical and Engineering Materials Division, Oak Ridge National Laboratory, Oak Ridge, \\ TN, 37831, USA
}

This manuscript has been authored by UT-Battelle, LLC under Contract No. DE-AC05-000R22725 with the U.S. Department of Energy. The United States Government retains and the publisher, by accepting the article for publication, acknowledges that the United States Government retains a non-exclusive, paid-up, irrevocable, world-wide license to publish or reproduce the published form of this manuscript, or allow others to do so, for United States Government purposes. The Department of Energy will provide public access to these results of federally sponsored research in accordance with the DOE Public Access Plan(http://energy.gov/downloads/doe-public-access-plan).

* Correspondence and requests for materials should be addressed to H.B. (email: beih@ornl.gov) 


\title{
Thermophysical properties of Ni-containing single-phase concentrated solid
}

\section{solution alloys}

\author{
K. Jin ${ }^{1}$, S. Mu ${ }^{1}$, K. An ${ }^{2}$, W. D. Porter ${ }^{1}$, G. D. Samolyuk ${ }^{1}$, G. M. Stocks ${ }^{1}$, and H. Bei ${ }^{1}$ \\ 1 Materials Science \& Technology Division, Oak Ridge National Laboratory, Oak Ridge, TN \\ 37831, USA \\ 2 Chemical and Engineering Materials Division, Oak Ridge National Laboratory, Oak Ridge, \\ TN, 37831, USA
}

\begin{abstract}
Temperature dependent thermophysical properties, including specific heat capacity, lattice thermal expansion, thermal diffusivity and conductivity, have been systematically studied in $\mathrm{Ni}$ and eight Ni-containing single-phase face-centered-cubic concentrated solid solution alloys, at elevated temperatures up to $1273 \mathrm{~K}$. The alloys have similar specific heat values of $0.4-0.5$ $\mathrm{J} \cdot \mathrm{g}^{-1} \cdot \mathrm{K}^{-1}$ at room temperature, but their temperature dependence varies greatly due to Curie and K-state transitions. The lattice, electronic, and magnetic contributions to the specific heat have been separated based on first-principles methods in $\mathrm{NiCo}, \mathrm{NiFe}, \mathrm{Ni}-20 \mathrm{Cr}$ and $\mathrm{NiCoFeCr}$. The alloys have similar thermal expansion behavior, with the exception that $\mathrm{NiFe}$ and $\mathrm{NiCoFe}$ have much lower thermal expansion coefficient in their ferromagnetic state due to magnetostriction effects. Calculations based on the quasi-harmonic approximation accurately predict the temperature dependent lattice parameter of $\mathrm{NiCo}$ and $\mathrm{NiFe}$ with less than $0.2 \%$ error, but underestimated that of $\mathrm{Ni}-20 \mathrm{Cr}$ by $1 \%$, compared to the values determined from neutron diffraction. All the alloys containing Cr have very similar thermal conductivity, which is much lower than that of $\mathrm{Ni}$ and the alloys without $\mathrm{Cr}$, due to the large magnetic disorder.
\end{abstract}

Keywords: Solid solution alloy, Thermophysical properties, Neutron diffraction, Chemical disorder 


\section{Introduction}

Thermophysical properties, e.g. specific heat capacity, coefficient of thermal expansion (CTE), and thermal conductivity, of transition metal alloys are of broad interest from both practical and scientific perspectives. For example, thermal expansion is an important concern in engineering applications of alloys, since the thermal stress and strain induced from temperature variations can greatly impact their high-temperature processing, a particularly critical factor when the alloys are used as part of composite material [1]. The Invar behavior observed in $\mathrm{Ni}-65 \mathrm{Fe}$ and other Ni-Fe-based alloys has long made understanding thermal expansion in these alloys an important scientific challenge [2]. The temperature dependent specific heat is not only a factor in determining thermal conductivity, but also an indicator of phase transitions such as magnetic phase and other order/disorder transitions [3-5]. Control of thermal conductivity is required in many practical applications, and both low (e.g. as thermal barriers and thermoelectric materials) and high (e.g. in cooling system, cladding tube in nuclear engineering, or other places where thermal shock needs to be prevented) values could be required depending on the application fields. Furthermore, thermal conductivity is a necessary parameter in theoretical studies of defect evolution under irradiation or energy deposition process, especially in the molecular dynamic simulations using a two-temperature model [6, 7].

The development of single-phase concentrated solid solution alloys (SP-CSA), including high entropy alloys, in the past decade [8-11] has gained significant interest due to their outstanding corrosion and wear resistance [12, 13], good weldability [14], exceptional mechanical properties [15-17], potential enhancement in radiation tolerance [18], and unique physical properties [19-22]. The large application potential of these alloys makes knowledge of their thermophysical properties highly desirable. In contrast to traditional alloys, which usually consist of one principal element and are typically multiphase, SP-CSA contain 2-5 (or even more) principal alloying elements [23] while condensing as single-phase solid-solutions on simple underlying lattices (e.g. single phase face-centered cubic, FCC). A significant feature of this novel class of alloys is that they offer new opportunities for tailoring materials properties through 
control of chemical complexity - changes in the number, type and concentration of alloying elements. Clearly, the notion of alloy design through control of chemical complexity not only greatly expands on traditional concepts of alloy design through microstructure modification but also raises challenges in understanding their intrinsic physical properties and opportunities of tuning them.

Recent studies have demonstrated that controlling compositional complexity of the Ni-containing FCC SP-CSAs can systematically tune their electrical and magnetic properties at low temperatures over wide ranges [19]. For example, the magnetic moment of $\mathrm{Cr}$ has been found anti-parallel to that of other elements when alloying with $\mathrm{Ni}, \mathrm{Co}$, and $\mathrm{Fe}$, which generates large magnetic disorder, causes strong scattering of electrons, and induces high (residual) electrical resistivity in the complex alloys [19]. In some of the alloys in this family, e.g. NiCoCr, unique quantum critical behavior has been observed, and its Curie temperature and transport properties can be dramatically changed with varying $\mathrm{Cr}$ concentration [21]. Although certain aspects of thermal properties have been measured by individual studies in various binary alloys in the early days (relevant references will be discussed in Section 3) and in some high entropy alloys, e.g. NiCoFeCrAl (note: usually not single-phase solid solution) and NiCoFeCrMn more recently $[24,25]$, systematic studies on the thermophysical properties in SP-CSA with varying compositional complexity at elevated temperature have been limited.

In the present study, a family of Ni-containing SP-CSAs from elemental Ni to quinary alloys, including equiatomic NiCo, $\mathrm{NiFe}$, NiCoFe, NiCoCr, NiCoFeCr, NiCoFeCrMn and NiCoFeCrPd, and non-equiatomic $\mathrm{Ni}-20 \mathrm{Cr}$ (since equiatomic $\mathrm{NiCr}$ is not a single-phase $\mathrm{FCC}$ solid solution [26]), were selected, and their thermophysical properties from 300 to $1273 \mathrm{~K}$ have been systematically investigated. The temperature dependent lattice parameter, constant pressure specific heat, and thermal diffusivity have been measured by neutron diffraction, differential scanning calorimeter (DSC), and laser flash technique, respectively. Moreover, the CTE and thermal conductivity of these alloys have then been derived and compared. First-principles calculations have been performed to evaluate the lattice parameter and the lattice contribution to 
the specific heat in the three binary alloys along with $\mathrm{NiCoFeCr}$.

\section{Experiments and methods}

\subsection{Materials}

Appropriate amounts of elemental (>99.9\% pure) $\mathrm{Ni}, \mathrm{Co}, \mathrm{Fe}, \mathrm{Cr}, \mathrm{Mn}$ and $\mathrm{Pd}$ metals (from Atlantic Equipment Engineers, NJ USA and Alfa Aesar, MA USA) were mixed by arc-melting. Each arc-melted button was flipped and re-melted five times before drop-casting the melt into a copper mold. The as-cast ingots were then homogenized at $1473 \mathrm{~K}$ for $24 \mathrm{~h}$, followed by water quenching. All the alloys have been experimentally determined to be single-phase solid solution with FCC crystal structure and the experimental details can be found elsewhere [26, 27]. Briefly, Fig. 1 shows the microstructure and X-ray diffraction results of the NiCoFeCrPd alloy as an example. The backscattered electron image only exhibits uniform grain structures and no secondary phase is observed. The Energy-dispersive X-ray spectroscopy (EDX) mapping further shows uniform elemental distribution. The X-ray diffraction pattern shown in Fig. 1c reveals only FCC peaks. These results confirm that the materials used in this study are single-phase solid solution alloys. The samples were then cut into various shapes using electro-discharge machining (EDM) to satisfy different characterization techniques.

\subsection{Characterizations}

The constant-pressure specific heats, $C_{p}$, were measured from 300 to $1000 \mathrm{~K}$ (to $1200 \mathrm{~K}$ for $\mathrm{NiCoFe}$ ) using a Netzsch $404 \mathrm{C}$ differential scanning calorimeter (DSC), following ASTM E 1269 using a sapphire standard. The samples were heated in a flowing argon atmosphere at a constant heating rate of $10 \mathrm{~K} / \mathrm{min}$. The samples experienced a full heating-cooling cycle before each measurement to rule out the exothermic reactions due to non-equilibrium cooling during the quench process $[4,28]$. For each sample, at least two measurements were performed and the heat capacity error of $5 \%$ was estimated.

Cylindrical samples with nominal diameter of $12 \mathrm{~mm}$ and length of $20 \mathrm{~mm}$ were used for in-situ neutron diffraction measurements, conducted on the VULCAN Engineering Materials Diffractometer at the Spallation Neutron Source, Oak Ridge National Laboratory (SNS, ORNL) 
[29]. The samples were heated by copper induction coils at a constant rate of $15 \mathrm{~K} / \mathrm{min}$, from 300 to $1273 \mathrm{~K}$, and the average lattice parameter and its changes upon heating were determined from the single peak fit of (111), (200), (220), and (311) peaks.

Thermal diffusivity was measured using a laser flash technique following ASTM E1461, using a Netzsch LFA 457 laser flash apparatus. Cylindrical sample disks with $12.7 \mathrm{~mm}$ in diameter and $\sim 3-6 \mathrm{~mm}$ in thickness were used for the measurements. Both flat surfaces of the sample disks were spray coated with colloidal graphite to increase the emissivity of the surfaces. Flowing argon was used as a purge gas to protect the samples from oxidation. The data were collected every $50 \mathrm{~K}$ from room temperature to $473 \mathrm{~K}$, and every $100 \mathrm{~K}$ up to $1273 \mathrm{~K}$ during heating. A few temperature points were re-collected during cooling, which showed about the same value as during heating.

\subsection{Calculation details}

First-principles calculations were performed using the projector augmented wave (PAW) method [30] implemented in the Vienna ab-initio simulation package (VASP) [31, 32]. The generalized gradient approximation (GGA) exchange-correlation functional in the formalism of PBE [33] was employed.. The energy cutoff for the plane-wave expansion was set to $400 \mathrm{eV}$, and a $\Gamma$-centered Monkhorst-Pack k-point grid was used for the Brillouin zone integration. A 32-atom special quasi-random structure (SQS) [34] was used to optimize the volume and all the atomic positions of considered alloys. The relaxation was performed using $1^{\text {st }}$-order Methfessel-Paxton method along with $6 \times 6 \times 6 \mathrm{k}$-point mesh, and the Hellmann-Feynman forces were converged to $0.005 \mathrm{eV} / \AA$.

Quasi-harmonic approximation (QHA) was employed for the thermal expansion, and the phonon calculations were performed using the linear response method implemented in VASP. Depending on the specific materials, the magnetism is incorporated differently. For NiFe and NiCo, the ferromagnetic ground state was used throughout the relaxation and the phonon calculations, ignoring the magnetostriction effects. On the contrary, $\mathrm{Ni}-20 \mathrm{Cr}$ has been proven to be nonmagnetic experimentally [35], therefore, magnetism of this system was prohibited in our 
calculations. To shed some light on the thermal behavior beyond the Curie temperature, several random collinear spin configurations were generated based on the SQS cells to model the phonon properties in the paramagnetic state. This is similar to the concept of the disorder local moment (DLM) state [36], but without sophisticated statistical averaging. Those DLM-like states are also applied to the $\mathrm{NiFeCoCr}$ alloys.

\section{Results and discussions}

\subsection{Specific heat}

The values of room temperature specific heat of all alloys tested are between 0.4 and 0.5 $\mathrm{J} \cdot \mathrm{g}^{-1} \cdot \mathrm{K}^{-1}$, as shown in Fig. $2 \mathrm{a}$ and $2 \mathrm{~b}$, while their temperature dependence varies significantly. For $\mathrm{Ni}, \mathrm{NiFe}$ and $\mathrm{NiCoFe}$, sharp peaks are observed at 628, 780, and $995 \mathrm{~K}$, respectively, which are attributed to the magnetic contribution to the specific heat, with peak temperatures indicating the Curie temperatures. The Curie temperature of $\mathrm{NiCo}$ is determined to be $1117 \mathrm{~K}$ by a separate DSC measurement (not shown here). These Curie points, as well as the values of specific heat of $\mathrm{Ni}$ and $\mathrm{NiFe}$, agree well with the literatures [37-40].

For $\mathrm{NiCoFeCrPd}$, a distinguishable but very small peak is observed at $374 \mathrm{~K}$, as shown in the inset of Fig. 2b, which agrees with the Curie temperature determined by magnetization measurements [19]. This much smaller peak, compared to that in the alloys mentioned above, indicates a smaller enthalpy of magnetic ordering in $\mathrm{NiCoFeCrPd}$, which might be related to its possible spin noncollinear ground state: the strong spin-orbit effects in $\mathrm{Pd}$, along with the exchange frustration in the alloy, facilitate the spin canting. The magnetic phase transition of the other studied alloys containing $\mathrm{Cr}$ appears below $300 \mathrm{~K}$ [19], and thus no corresponding peaks were observed in this study.

Other than the Curie transition, endothermic reactions, shown as an abrupt increase in specific heat, were observed for all the alloys containing $\mathrm{Cr}$, as well as for $\mathrm{NiCoFe}$, within a transition temperature window of $750-950 \mathrm{~K}$. All these curves were reproduced at least twice, the $C_{p}-\mathrm{T}$ curves from the second heating-cooling cycles are identical to those from the first cycles, and the 
possible oxidation issue is then excluded.

Similar transitions regarding specific heat in the same temperature window in the alloys containing $\mathrm{Ni}$ and $\mathrm{Cr}$ have been reported, not only for the commercial alloys such as 617 [41], 600 [4], and Ni-Cr-Mo [42] systems, but also for the model alloys such as Ni-20Cr [41] and $\mathrm{Ni}-\mathrm{Fe}-\mathrm{Cr}$ [43]. Other thermal anomalies in physical (e.g. electrical resistivity) and mechanical properties (e.g. creep) in the Ni-Cr- containing alloys on the Ni-rich side have also been commonly observed $[44,45]$. These unique behaviors have been generally termed as "komplex-" or K-state [46], the origin of which has been extensively studied over the decades experimentally and theoretically but still not yet fully clarified. A few of these studies attributed the K-state mainly to the development of long-range order, e.g. dispersed ordered $\mathrm{Ni}_{2} \mathrm{Cr}$ domains in a random or disordered matrix [4, 44]. In contrast, many others believed that only short-range order occurs in the alloys with Cr concentration below 25 at\% [45, 47]. Short-range order in this family of alloys has been measured repeatedly using diffuse neutron [48, 49] and X-ray [50] scattering and theoretically studied by first-principles calculations [51].

The formation of a long-range ordered $\mathrm{Ni}_{2} \mathrm{Cr}$ phase has been reported in $\mathrm{Ni}-\mathrm{Cr}$ alloys after long-term (hundreds to thousands of hours) annealing at temperatures below 500-600 ${ }^{\circ} \mathrm{C}[44,52]$. The evidence of such long-range ordering in neutron diffraction patterns is the appearance of superlattice peaks [52], corresponding to a $\mathrm{Mo}_{2} \mathrm{Pt}$-type orthorhombic crystal structure. In the current study, however, the starting materials were water-quenched and the heating time during measurements were limited to a few hours. Neutron diffraction patterns from these materials do not show any sign of superlattice peaks. For example, Fig. 3 shows the representative neutron diffraction patterns of $\mathrm{Ni}-20 \mathrm{Cr}$ and $\mathrm{NiCoFeCrMn}$ alloys that only exhibit FCC peaks, indicating no detectable long-range ordered $\mathrm{Ni}_{2} \mathrm{Cr}$ phase. In addition, with repeated measurements on each sample, the heat absorption in the transitions remained the same between each test cycle. Furthermore, a single crystalline $\mathrm{Ni}-20 \mathrm{Cr}$ sample grown using the floating-zone directional solidification method was also tested for comparison, and exhibited about the same $C_{p}-\mathrm{T}$ curve as the quenched polycrystalline samples. Therefore, the transitions in our specific heat 
measurements are believed to be attributed to the short-range order/disorder transitions, while the detailed mechanism requires further investigations [41]. It needs to be emphasized that all the alloys containing $\mathrm{Cr}$ selected in this study exhibit a similar transition, from binary to quinary with $\mathrm{Cr}$ concentration from 20 to 33 at\%, which implies that all Cr-contained alloys might exhibit short-range order. Interestingly, for the alloys without $\mathrm{Cr}$ (meaning that $\mathrm{Ni}-\mathrm{Cr}$ related short range order is not possible), only NiCoFe alloy exhibits a similar transition around 750-800 K. Unfortunately, the current experiments and state of knowledge do not reveal the nature of this transition, a situation that will be revisited in future studies.

The specific heat of the Invar-type alloys (Ni-Fe-based) has been measured over the past decades with controversial results. Bendick et al [53]. observed an anomalous maximum at $\sim 500 \mathrm{~K}$ in addition to the one at the Curie temperature for $\mathrm{Ni}-50 \mathrm{Fe}$, and attributed this behavior to a Schottky-type anomaly assuming a two-level model. However, this anomaly was not observed by Tanji et al [54]. The studies by Hausch [55] on Ni-44Fe and by Orehotsky et al. [37] on $\mathrm{Ni}-55 \mathrm{Fe}$ also showed no additional peaks in this temperature range. Here our measurement disagrees with Bendick's but supports the results of others that no additional maximum exists at $\sim 500 \mathrm{~K}$.

The lattice contribution to the specific heats of $\mathrm{NiCo}, \mathrm{NiFe}, \mathrm{Ni}-20 \mathrm{Cr}$, and $\mathrm{NiCoFeCr}$ has been calculated from first-principles. Since specific heat consists of lattice, electronic, and magnetic components, all the calculated results are smaller than the experimental results. The contribution from electrons is relatively small and usually approximately expressed as $C_{e}=\gamma T$, where $\gamma$ is called the electronic specific heat coefficient [5]. The values of $\gamma$ for NiCo and NiFe are both $\sim 7.2 \times 10^{-5} \mathrm{~J} \cdot \mathrm{g}^{-1} \cdot \mathrm{K}^{-2}$ [37] and the addition of the lattice and electronic contributions of these two alloys are plotted in Fig. 4a; the magnetic contributions are derived by subtracting these two contributions from the measured results. A lambda shape is observed as expected. The magnetic contribution is negligible in the low temperature regime, increases when approaching the Curie temperature, and vanishes at higher temperatures. For $\mathrm{Ni}-20 \mathrm{Cr}$ and $\mathrm{NiCoFeCr}$, the electronic component can be derived from the difference between the experimental value and the calculated 
lattice component, since the measurements were taken above the Curie temperature, as shown in Fig. 4b. The derived electronic specific heats of these two alloys are similar and are both linear with temperature; the estimated $\gamma$ values are both $6.3 \times 10^{-5} \mathrm{~J} \cdot \mathrm{g}^{-1} \cdot \mathrm{K}^{-2}$, which is similar to that of $\mathrm{NiCo}$ and $\mathrm{NiFe}$. Due to the unclear nature of the K-state transition, the contribution from each component after $800 \mathrm{~K}$ in $\mathrm{Ni}-20 \mathrm{Cr}$ and $\mathrm{NiCoFeCr}$ was not quantified.

\subsection{Lattice parameter}

The temperature-dependent lattice parameter is shown in Fig. 5a, from 300 to $1273 \mathrm{~K}$, according to which the theoretical density as a function of temperature is then calculated. The lattice parameters are derived from the (200), (220), (111), and (311) peaks. An empirical formula in exponential form

$$
L=L_{0}+b T+c[\operatorname{Exp}(-T / d)-1]
$$

where $L$ and $L_{0}$ are the lattice parameters at temperature $T$ and $0 \mathrm{~K}$, respectively; $b, c$, and $d$ are fitting parameters, can well describe the temperature dependence of lattice parameter for all these materials except $\mathrm{NiFe}$ and $\mathrm{NiCoFe}$, in which magnetostriction effects may cause a significant change in thermal expansion [56]. Spline estimation is thus used to fit the curves for $\mathrm{NiFe}$ and NiCoFe. A similar form of Eq. 1, in which $b, c$, and $d$ are correlated, has been used to describe the thermal expansion for some FCC steels [57] and NiCoFeCrMn [24] measured by dilatometer. Here, however, they need to be determined independently to reasonably fit the full set of alloys. All the fitting curves are shown as the solid lines in Fig. 5a.

The room temperature lattice parameters determined from our neutron diffraction measurement are in good agreement to the previous results based on X-ray diffraction [58]. In addition, the calculated densities at room temperature have little difference as compared with the experimental value; thus the deduced temperature-dependent density, $\rho$, is used in the calculation of thermal conductivity.

For the three binary alloys, $\mathrm{NiCo}, \mathrm{NiFe}, \mathrm{Ni}-20 \mathrm{Cr}$, the temperature dependent lattice parameters 
were calculated from first-principles, as shown in comparison with experiments in Fig. 5b. The calculated lattice parameters in $\mathrm{NiFe}$ and $\mathrm{NiCo}$ agree with the experimental values with an error less than $\sim 0.2 \%$. On the contrary, for $\mathrm{Cr}$ containing alloys, $\mathrm{PBE}$ underestimate the lattice parameter of the supercells by $1 \%$. This underestimation of the lattice spacing can be traced back to the antiferromagnetic spin alignment on Mn; GGA is less successful correcting the LDA overbinding problem for antiferromagnetic than ferromagnetic $3 d$ metals, as shown in antiferromagnetic $\alpha-\mathrm{Mn}$, bcc-Cr, and $\gamma-\mathrm{Fe}$ [59].

The thermal expansion effect was evaluated by QHA, which is valid for systems without strong anharmonicity. Our cases presumably qualify due to the small mass disorder. As found in Fig. 5b, the calculated lattice parameter of $\mathrm{NiFe}$ increases faster with temperature than the experimental result. This is partially because QHA neglects the possible spin-phonon coupling, and thus it cannot capture the magnetostriction effect at elevated temperature. In addition, the thermal electronic effect was not included in the treatment since it is usually small compared with the vibrational effect. The entropy from the magnetism is also neglected in the free energy. Nevertheless, overall, this crude approximation provides reasonable predictions on lattice parameters and heat capacity.

The temperature dependent lattice parameter can provide information on the coefficient of thermal expansion, although some fine features, such as the peak of CTE right at the Curie temperature in $\mathrm{Ni}$ [60], cannot be captured. This is mainly due to the fact that the measured lattice parameters are bulk averaged over the temperature interval of $15 \mathrm{~K}$, which is too large to properly capture such features [1]. Here the derived CTE value [61] of

$\alpha=(1 / L) \mathrm{d} L / \mathrm{d} T$

is shown in Fig. 6. The CTE values of $\mathrm{Ni}, \mathrm{NiCo}, \mathrm{NiCoCr}, \mathrm{NiCoFeCr}$, and $\mathrm{Ni}-20 \mathrm{Cr}$ are very similar, while that of NiCoFeCrMn is slightly higher but follows the same trend. In contrast, the CTE of $\mathrm{NiFe}$ and $\mathrm{NiCoFe}$ are apparently lower in their ferromagnetic state due to the spontaneous volume magnetostriction (although not close to zero as the case of typical Invar 
alloys such as $\mathrm{Ni}-65 \mathrm{Fe}[2])$, and sharply increase beyond the Curie point. In the paramagnetic regime, the $\mathrm{CTE}$ of $\mathrm{NiFe}$ and $\mathrm{NiCoFe}$ become similar to the other alloys. Such temperature dependence of CTE in NiFe is similar to what has been observed in the dilatometer measurements [56].

\subsection{Thermal diffusivity and thermal conductivity}

The thermal diffusivity, $D$, and the derived [25] thermal conductivity using

$\kappa=D \rho C_{p}$

are shown in Fig. 7a and 7b, respectively. Below the Curie temperature, the thermal diffusivity and conductivity of the alloys that do not contain $\mathrm{Cr}$ are significantly larger than those of the alloys containing $\mathrm{Cr}$, although smaller than those of pure $\mathrm{Ni}$. This is mainly attributed to the fact that the electrical resistivity of the alloys without $\mathrm{Cr}$ is much smaller than that of $\mathrm{Cr}$ containing alloys, giving them larger electronic thermal conductivity [19]. The four systems that do not contain $\mathrm{Cr}$ also have negative coefficient of temperature dependence of thermal diffusivity/conductivity below the Curie temperature that is attributed to the fast increase of electrical resistivity according to the Wiedemann-Franz relationship

$\kappa_{e}=L T / \rho$,

where $\kappa_{e}$ is the electronic thermal conductivity, $\rho$ is the electrical resistivity, and $L$ is the Lorentz constant [62].

A minimum in thermal diffusivity can be clearly seen around the Curie temperatures of the four Cr-free samples although the temperature interval is somewhat large, after which the thermal diffusivities increase with increasing temperature. Such minima have been commonly observed in magnetic metals and alloys in the past (e.g. Ni [63], Fe [64], Gd [65], Ni-Fe [62], and Ni-Cu [62]). One of the simple interpretations is that the electrical resistivity induced from spin fluctuation becomes saturated above the Curie temperature, leading to a sudden slow-down in the increase of electrical resistivity as a function of temperature which, according to the 
Widemann-Franz relationship, causes the increase of thermal conductivity above Curie temperature [66]. However, it should be noted that such critical point anomalies in thermal conductivity might also be somewhat attributed to the contribution from the lattice component through spin-lattice coupling [63]. While non-trivial this possibility will not be discussed in the present work.

For the paramagnetic and non-magnetic Cr-containing alloys the thermal diffusivity and thermal conductivity increase with increasing temperature over the entire temperature range. This is mainly because these alloys have large residual resistivity (the resistivity at $0 \mathrm{~K}$ ), and very low temperature dependence of the resistivity. Such invariant electrical resistivity as a function of temperature makes their electronic thermal conductivity increase almost linearly with temperature [19].

\section{Summary and conclusions}

In summary, the temperature dependence of specific heat, lattice parameter, and thermal diffusivity has been measured in a family of Ni-containing single phase concentrated solid solution alloys at elevated temperature. The thermal conductivity and thermal expansion coefficient of these alloys have been derived, and their Curie temperatures have been determined. All these alloys have similar specific heat at room temperature, but their temperature dependence varies a great deal depending on the magnetic state and possible short-range order-disorder transitions. The lattice contribution of the specific heat has been calculated from first-principles, and the electronic and magnetic contributions have been derived accordingly. The neutron-diffraction-determined lattice parameters, in order from small to large at room temperature are: $\mathrm{Ni}, \mathrm{NiCo}, \mathrm{Ni}-20 \mathrm{Cr}, \mathrm{NiCoCr}, \mathrm{NiCoFeCr}, \mathrm{NiCoFe}, \mathrm{NiFe}, \mathrm{NiCoFeCrMn}$, and $\mathrm{NiCoFeCrPd}$ (the latter being determined by X-ray diffraction). The QHA-based calculations provide a good estimate of the lattice parameters of $\mathrm{NiCo}$ and $\mathrm{NiFe}$, within $0.2 \%$ error, but underestimate that of $\mathrm{Ni}-20 \mathrm{Cr}$ by $\sim 1 \%$. All these alloys have similar thermal expansion coefficients, except that $\mathrm{NiFe}$ and $\mathrm{NiCoFe}$ show strong magnetostriction effects at their 
ferromagnetic state, and NiCoFeCrMn shows slightly higher CTE than that of the others. The thermal transport properties can be grouped whether or not they contain the element Cr. Nickel and alloys without $\mathrm{Cr}$ show larger thermal diffusivity and conductivity, have negative temperature coefficients below their Curie points, and have positive temperature coefficients at higher temperatures. All the alloys containing $\mathrm{Cr}$ have very similar thermal diffusivity and thermal conductivity, which increase with increasing temperature from room temperature to at least $1000 \mathrm{~K}$.

\section{Acknowledgment}

This work was supported as part of the Energy Dissipation to Defect Evolution (EDDE), an Energy Frontier Research Center funded by the U.S. Department of Energy, Office of Science, Basic Energy Sciences. A portion of this research used resources at Spallation Neutron Source, a DOE Office of Science User Facility operated by the Oak Ridge National Laboratory. 


\section{References}

[1] J.D. James, J.A. Spittle, S.G.R. Brown, R.W. Evans. A review of measurement techniques for the thermal expansion coefficient of metals and alloys at elevated temperatures, Meas. Sci. Technol. 12 (2001) R1-R15.

[2] M. van Schilfgaarde, I.A. Abrikosov, B. Johansson. Origin of the Invar effect in iron-nickel alloys, Nature 400 (1999) 46-49.

[3] D.M. Kroeger, C.C. Koch, J.O. Scarbrough, C.G. McKamey. Effects of short-range order on electronic properties of Zr-Ni glasses as seen from low-temperature specific heat, Phys. Rev. B 29 (1984) 1199-1208.

[4] S. Kim, I.H. Kuk, J.S. Kim. Order-disorder reaction in Alloy 600, Mater. Sci. Eng. A 279 (2000) 142-148.

[5] R.E. Pawel, E.E. Stansbury. Specific heat contributions of the ferromagnetic transition in nickel and nickel-copper alloys, J. Phys. Chem. Solid. 26 (1965) 757-765.

[6] M. Caro, L.K. Béland, G.D. Samolyuk, R.E. Stoller, A. Caro. Lattice thermal conductivity of multi-component alloys, J. Alloy. Comp. 648 (2015) 408-413.

[7] M. Toulemonde, W. Assmann, C. Dufour, A. Meftah, F. Studer, C. Trautmann. Experimental phenomena and thermal spike model description of ion tracks in amorphisable inorganic insulators, Mat. Fys. Medd. K. Dan. Vidensk. Selsk. 52 (2006) 263-292.

[8] B. Cantor, I.T.H. Chang, P. Knight, A.J.B. Vincent. Microstructural development in equiatomic multicomponent alloys, Mater. Sci. Eng. A 375-377 (2004) 213-218.

[9] O.N. Senkov, G.B. Wilks, J.M. Scott, D.B. Miracle. Mechanical properties of $\mathrm{Nb}_{25} \mathrm{Mo}_{25} \mathrm{Ta}_{25} \mathrm{~W}_{25}$ and $\mathrm{V}_{20} \mathrm{Nb}_{20} \mathrm{Mo}_{20} \mathrm{Ta}_{20} \mathrm{~W}_{20}$ refractory high entropy alloys, Intermetallics 19 (2011) 698-706.

[10] M.S. Lucas, G.B. Wilks, L. Mauger, J.A. Muñoz, O.N. Senkov, E. Michel, J. Horwath, S.L. Semiatin, M.B. Stone, D.L. Abernathy, E. Karapetrova. Absence of long-range chemical ordering in equimolar FeCoCrNi, Appl. Phys. Lett. 100 (2012) 251907.

[11] F. Tancret, I. Toda-Caraballo, E. Menou, P.E.J. Rivera Díaz-Del-Castillo. Designing high entropy alloys employing thermodynamics and Gaussian process statistical analysis, Mater. Des. 115 (2017) 486-497.

[12] Y. Zhang, T. Zuo, Z. Tang, M.C. Gao, K.A. Dahmen, P.K. Liaw, Z. Lu. Microstructures and properties of high-entropy alloys, Prog. Mater. Sci. 61 (2014) 1-93.

[13] Z. Zhang, E. Axinte, W. Ge, C. Shang, Y. Wang. Microstructure, mechanical properties and corrosion resistance of CuZrY/Al, Ti, Hf series high-entropy alloys, Mater. \& Des. 108 (2016) 106-113.

[14] Z. Wu, S.A. David, Z. Feng, H. Bei. Weldability of a high entropy CrMnFeCoNi alloy, Scripta Mater. 124 (2016) 81-85.

[15] B. Gludovatz, A. Hohenwarter, D. Catoor, E.H. Chang, E.P. George, R.O. Ritchie. A fracture-resistant high-entropy alloy for cryogenic applications, Science 345 (2014) 1153-1158.

[16] B. Gludovatz, A. Hohenwarter, K.V. Thurston, H. Bei, Z. Wu, E.P. George, R.O. Ritchie. Exceptional damage-tolerance of a medium-entropy alloy CrCoNi at cryogenic temperatures, Nat. Commun. 7 (2016) 10602.

[17] Y.D. Wu, Y.H. Cai, X.H. Chen, T. Wang, J.J. Si, L. Wang, Y.D. Wang, X.D. Hui. Phase composition and solid solution strengthening effect in TiZrNbMoV high-entropy alloys, Mater. \& Des. 83 (2015) 651-660.

[18] K. Jin, C. Lu, L.M. Wang, J. Qu, W.J. Weber, Y. Zhang, H. Bei. Effects of compositional complexity on the ion-irradiation induced swelling and hardening in Ni-containing equiatomic alloys, Scripta Mater. 119 (2016) 65-70.

[19] K. Jin, B.C. Sales, G.M. Stocks, G.D. Samolyuk, M. Daene, W.J. Weber, Y. Zhang, H. Bei. Tailoring the physical properties of Ni-based single-phase equiatomic alloys by modifying the chemical complexity, Sci. Rep. 6 
(2016) 20159.

[20] Y. Zhang, G.M. Stocks, K. Jin, C. Lu, H. Bei, B.C. Sales, L. Wang, L.K. Beland, R.E. Stoller, G.D. Samolyuk, M. Caro, A. Caro, W.J. Weber. Influence of chemical disorder on energy dissipation and defect evolution in concentrated solid solution alloys, Nat. Commun. 6 (2015) 8736.

[21] B.C. Sales, K. Jin, H. Bei, G.M. Stocks, G.D. Samolyuk, A.F. May, M.A. McGuire. Quantum Critical Behavior in a Concentrated Ternary Solid Solution, Sci. Rep. 6 (2016) 26179.

[22] K.Y. Tsai, M.H. Tsai, J.W. Yeh. Sluggish diffusion in Co-Cr-Fe-Mn-Ni high-entropy alloys, Acta Mater. 61 (2013) 4887-4897.

[23] J.W. Yeh, S.K. Chen, S.J. Lin, J.Y. Gan, T.S. Chin, T.T. Shun, C.H. Tsau, S.Y. Chang. Nanostructured high-entropy alloys with multiple principal elements: novel alloy design concepts and outcomes, Adv. Eng. Mater. 6 (2004) 299-303.

[24] G. Laplanche, P. Gadaud, O. Horst, F. Otto, G. Eggeler, E.P. George. Temperature dependencies of the elastic moduli and thermal expansion coefficient of an equiatomic, single-phase CoCrFeMnNi high-entropy alloy, J. Alloy. Compound. 623 (2015) 348-353.

[25] H. Chou, Y. Chang, S. Chen, J. Yeh. Microstructure, thermophysical and electrical properties in $\mathrm{Al}_{\mathrm{x}} \mathrm{CoCrFeNi}$ $(0 \leq x \leq 2)$ high-entropy alloys, Mater. Sci. Eng. B 163 (2009) 184-189.

[26] Z. Wu, H. Bei, F. Otto, G.M. Pharr, E.P. George. Recovery, recrystallization, grain growth and phase stability of a family of FCC-structured multi-component equiatomic solid solution alloys, Intermetallics 46 (2014) 131-140.

[27] F. Otto, A. Dlouhý, C. Somsen, H. Bei, G. Eggeler, E.P. George. The influences of temperature and microstructure on the tensile properties of a CoCrFeMnNi high-entropy alloy, Acta Mater. 61 (2013) 5743-5755.

[28] M. Hirabayashi, M. Koiwa, K. Tanaka, T. Tadaki, T. Saburi, S. Nenno, H. Nishiyama. An experimental study on the ordered alloy $\mathrm{Ni}_{2} \mathrm{Cr}$, Trans. JPN. I. Met. 10 (1969) 365.

[29] K. An, H.D. Skorpenske, A.D. Stoica, D. Ma, X. Wang, E. Cakmak. First In Situ Lattice Strains Measurements Under Load at VULCAN, Metall. Mater. Trans. A 42 (2010) 95-99.

[30] P.E. Blöchl. Projector augmented-wave method, Phys. Rev. B 50 (1994) 17953-17979.

[31] G. Kresse, J. Hafner. Ab initiomolecular dynamics for open-shell transition metals, Phys. Rev. B 48 (1993) 13115-13118.

[32] G. Kresse, J. Furthmüller. Efficient iterative schemes forab initiototal-energy calculations using a plane-wave basis set, Phys. Rev. B 54 (1996) 11169-11186.

[33] J.P. Perdew, K. Burke, M. Ernzerhof. Generalized Gradient Approximation Made Simple, Phys. Rev. Lett. 77 (1996) 3865-3868.

[34] A. Zunger, S. Wei, L.G. Ferreira, J.E. Bernard. Special quasirandom structures, Phys. Rev. Lett. 65 (1990) 353-356.

[35] M.J. Besnus, Y. Gottehrer, G. Munschy. Magnetic properties of Ni-Cr alloys, Phys. Status Solidi b 49 (1972) 597-607.

[36] B.L. Gyorffyt, A.J. Pindors, J. Stauntonll, G.M. Stocks, H. Winter. A first-principles theory of ferromagnetic phase transitions in metals, J. Phys. F: Met. Phys. 15 (1985) 1337.

[37] J. Orehotsky, K. Schröder. Specific Heat of Nickel-Iron and Nickel-Cobalt Alloys between $600^{\circ} \mathrm{K}$ and $1500^{\circ} \mathrm{K}$, Phys. Cond. Matter 17 (1973) 37-53.

[38] P. Handler, D.E. Mapother, M. Rayl. AC Measurement of the Heat Capacity of Nickel Near its Critical Point, Phys. Rev. Lett. 19 (1967) 356-358. 
[39] D.L. Connelly, J.S. Loomis, D.E. Mapother. Specific Heat of Nickel near the Curie Temperature, Phys. Rev. B 3 (1971) 924-934.

[40] T.C. Pilkington, J.L. Artley, F.T. Wooten. Prediction of Curie Temperatures in Ternary Fe-Co-Ni Alloys. II, J. Appl. Phys. 35 (1964) 3493.

[41] B.H. Rabin, W.D. Swank, R.N. Wright. Thermophysical properties of Alloy 617 from $25^{\circ} \mathrm{C}$ to $1000^{\circ} \mathrm{C}$, Nucl. Eng. Des. 262 (2013) 72-80.

[42] B. Wilthan, H. Reschab, R. Tanzer, W. Schützenhöfer, G. Pottlacher. Thermophysical Properties of a Chromium-Nickel-Molybdenum Steel in the Solid and Liquid Phases, Int. J. Thermophys 29 (2007) 434-444.

[43] K.G. Binnatov, A.A. Katsnel'son, Y.L. Rodionov. Atomic ordering in Ni-Fe-Cr solid solutions, Soviet Phys. J. 18 (1975) 397-398.

[44] A. Marucco, B. Nath. Effects of Ordering on the Properties of Ni-Cr Alloys, J. Mater. Sci. 23 (1988) 2107-2114.

[45] E.E. Stansbury, C.R. Brooks, T.L. Arledge. Specific-Heat Anomalies in Solid Solutions of Chromium and Molybdenum in Nickel - Evidence for Short-Range Order, J. Inst. Metal. 94 (1966) 136-138.

[46] H. Thomas. Über Widerstandslegierungen, Z. F Physik 129 (1951) 219-232.

[47] P. Nash. The Cr-Ni (chromium-nickel) system, Bull. Alloy Phase Diagr. 7 (1986) 466-476.

[48] B. Schonfeld, F. Klaiber, G. Kostorz, U. Zaune, G. Mcintyre. Diffuse Neutron-Scattering and Short-Range Order in Ni-19.4 at Percent Cr, Scripta Metal. 20 (1986) 385-387.

[49] B. Schönfeld, L. Reinhard, G. Kostorz, W. Bührer. Short-Range Order and Atomic Displacements in Ni-20 at\% Cr Single Crystals, Phys. Status Solidi B 148 (1988) 457-471.

[50] B. Schönfeld, G.E. Ice, C.J. Sparks, H.G. Haubold, W. Schweika, L.B. Shaffer. X-ray study of diffuse scattering in Ni-20 at\% Cr, Phys. Status Solidi B 183 (1994) 79-95.

[51] M. Rahaman, B. Johansson, A.V. Ruban. First-principles study of atomic ordering in fcc Ni-Cr alloys, Phys. Rev. B 89 (2014) 064103.

[52] S. Kim. Order-disorder reaction in $\mathrm{Ni}_{2} \mathrm{Cr}$ alloy, Transactions of the Korean Nuclear Society Autumn Meeting Gyeongju, Korea, October 29-30 (2009) 313.

[53] W. Bendick, H.H. Ettwig, W. Pepperhoff. Anomalies in Specific-Heat and Thermal-Expansion of Fcc Iron-Alloys, J. Phys. F Metal Phys. 8 (1978) 2525-2534.

[54] Y. Tanji, H. Asano, H. Moriya. Specific Heats of Fe-Ni (fcc) Alloys at High Temperature, Sci. Rep. Res. Inst., Tohoku University. Ser. A, Phys., Chem. Metall. 24 (1972) 205-217.

[55] G. Hausch. High-Temperature Specific-Heat of Feni and Fept Invar-Alloys, J Magn Magn Mater 92 (1990) 87-91.

[56] Y. Tanji. Thermal Expansion Coefficient and Spontaneous Volume Magnetostriction of Fe-Ni (fcc) Alloys, J. Phys. Soc. Japan 31 (1971) 1366-1373.

[57] S.M.C. van Bohemen. The nonlinear lattice expansion of iron alloys in the range 100-1600K, Scripta Mater. 69 (2013) 315-318.

[58] Z. Wu. Temperature and alloying effects on the mechanical properties of equiatomic FCC solid solution alloys., $\mathrm{Ph}$. D Dissertation in Materials Science and Engineering, Knoxville (2014).

[59] D. Hobbs, J. Hafner, D. Spišák. Understanding the complex metallic element Mn. I.Crystalline and noncollinear magnetic structure of $\alpha$-Mn, Phys. Rev. B 68 (2003) 014407.

[60] T.G. Kollie. Measurement of the thermal-expansion coefficient of nickel from 300 to $1000 \mathrm{~K}$ and determination 
of the power-law constants near the Curie temperature, Phys. Rev. B 16 (1977) 4872-4881.

[61] D. Yu, K. An, X. Chen, H. Bei. Phase-specific deformation behavior of a NiAl-Cr(Mo) lamellar composite under thermal and mechanical loads, J. Alloy Comp. 656 (2016) 481-490.

[62] C.Y. Ho, M.W. Ackerman, K.Y. Wu, S.G. Oh, T.N. Havill. Thermal conductivity of ten selected binary alloy systems, J. Phys. Chem. Ref. Data 7 (1978) 959-1177.

[63] R.W. Powell, R.P. Tye, M.J. Hickman. The thermal conductivity of nickel, Int. J. Heat .Mass Tran. 8 (1965) 679-688.

[64] M.D. Lanchbury, N.H. Saunders. Critical behaviour in the transport properties of pure iron, J. Phys. F Metal Phys. 6 (1976) 1967-1977.

[65] C. Glorieux, J. Thoen, G. Bednarz, M.A. White, D.J.W. Geldart. Photoacoustic investigation of the temperature and magnetic-field dependence of the specific-heat capacity and thermal conductivity near the Curie point of gadolinium, Phys. Rev. B 52 (1995) 12770-12778.

[66] H. Ebert, S. Mankovsky, K. Chadova, S. Polesya, J. Minár, D. Ködderitzsch. Calculating linear-response functions for finite temperatures on the basis of the alloy analogy model, Phys. Rev. B 91 (2015) 165132. 


\section{Figures}

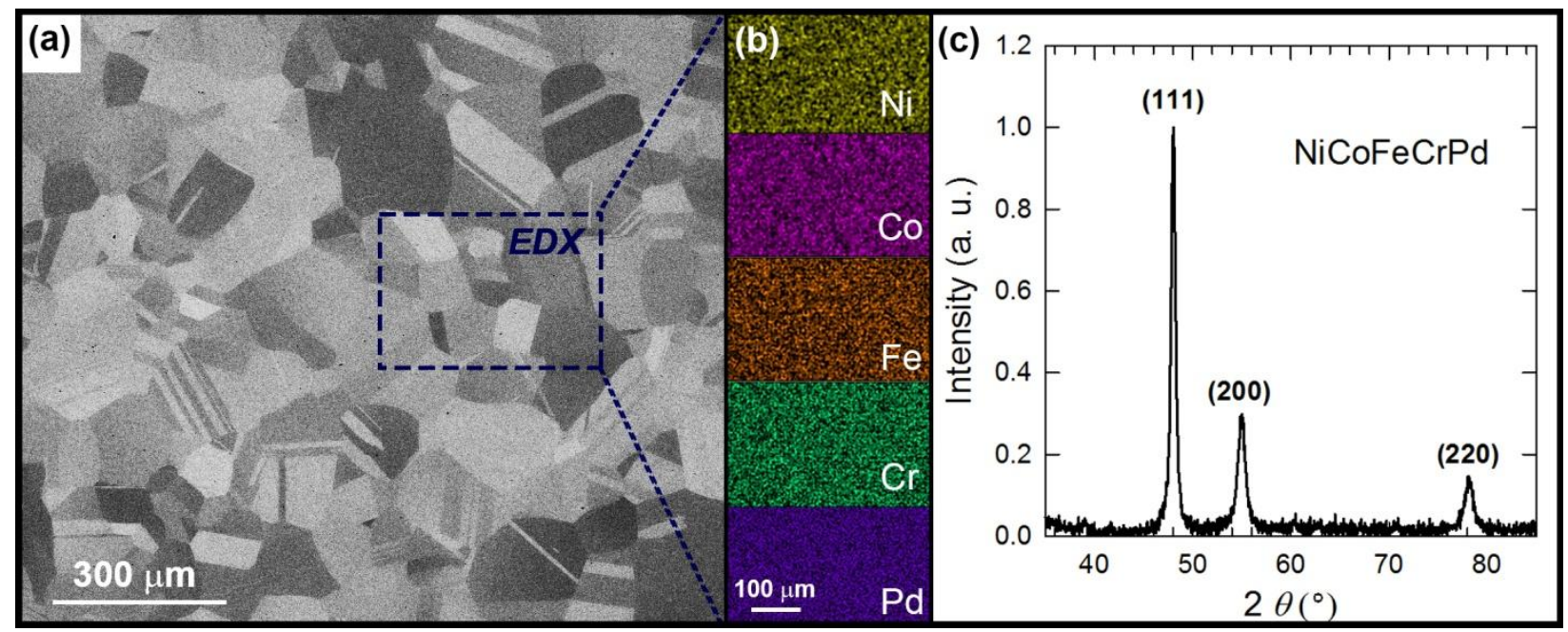

Fig. 1. (a) Backscattered electron images of the microstructures of $\mathrm{NiCoFeCrPd}$ after homogenized at $1473 \mathrm{~K}$ for $24 \mathrm{~h}$. The square inset indicates the region where the EDX scan was performed (shown in b). (c) X-ray diffraction pattern of NiCoFeCrPd, in which only FCC peaks appear. 

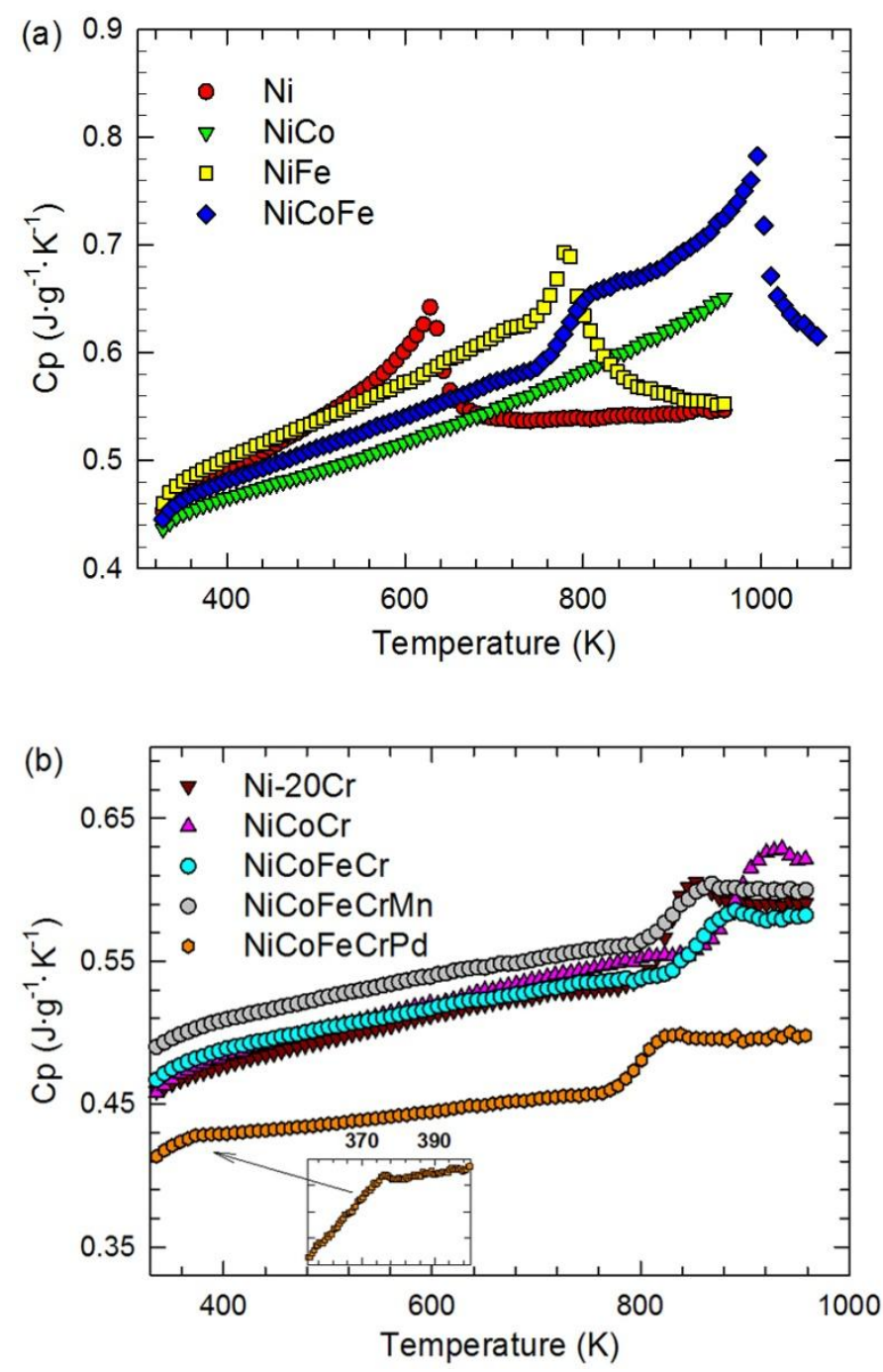

Fig. 2. Temperature dependent constant pressure specific heat of (a) $\mathrm{Ni}, \mathrm{NiCo}, \mathrm{NiFe}$, and $\mathrm{NiCoFe}$, which do not contain $\mathrm{Cr}$, and (b) Ni-20Cr, NiCoCr, NiCoFeCr, NiCoFeCrMn and NiCoFeCrPd. The inset of (b) is the enlarged plot for NiCoFeCrPd showing a small peak at $374 \mathrm{~K}$. 

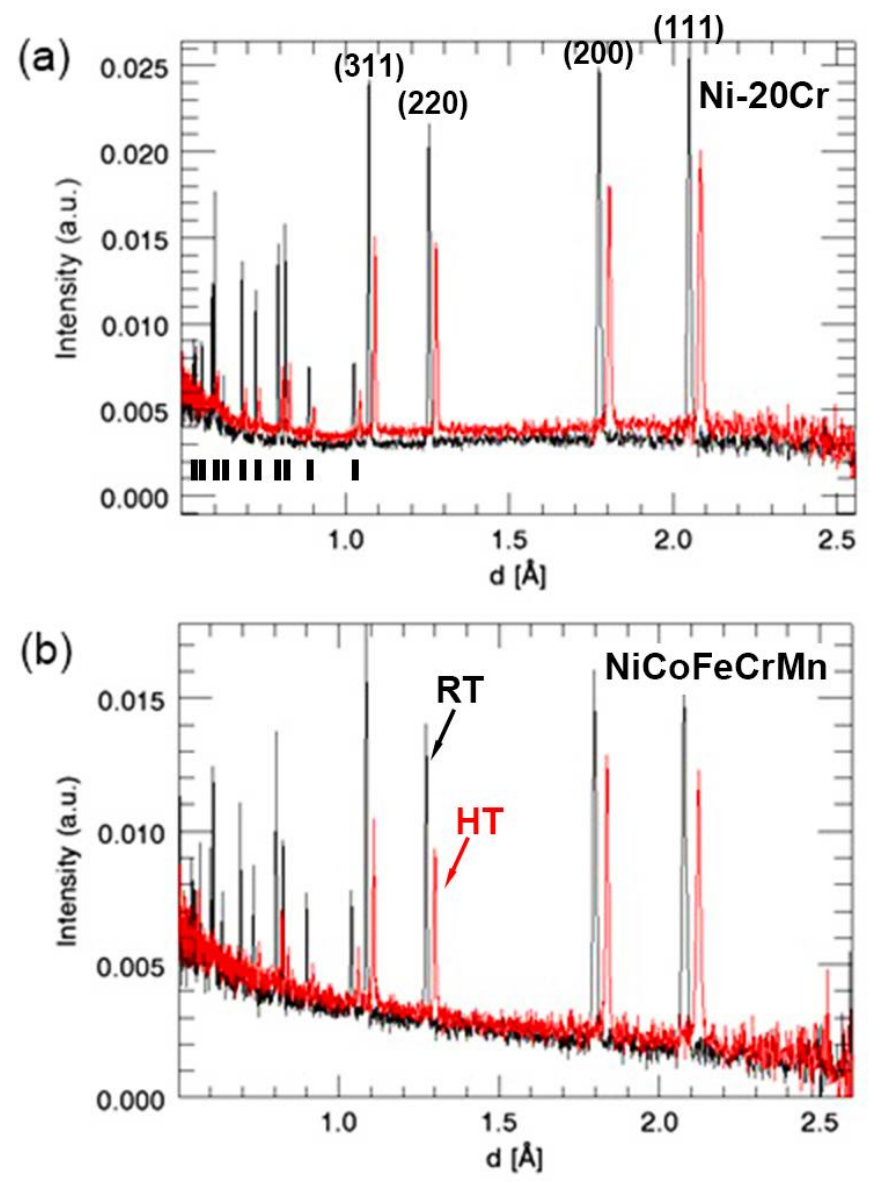

Fig. 3. Neutron diffraction pattern of (a) Ni-20Cr and (b) NiCoFeCrMn at room temperature (RT) and high temperature (HT). The patterns have been indexed, and the low-index peaks are labeled in (a). Only FCC peaks appear. 

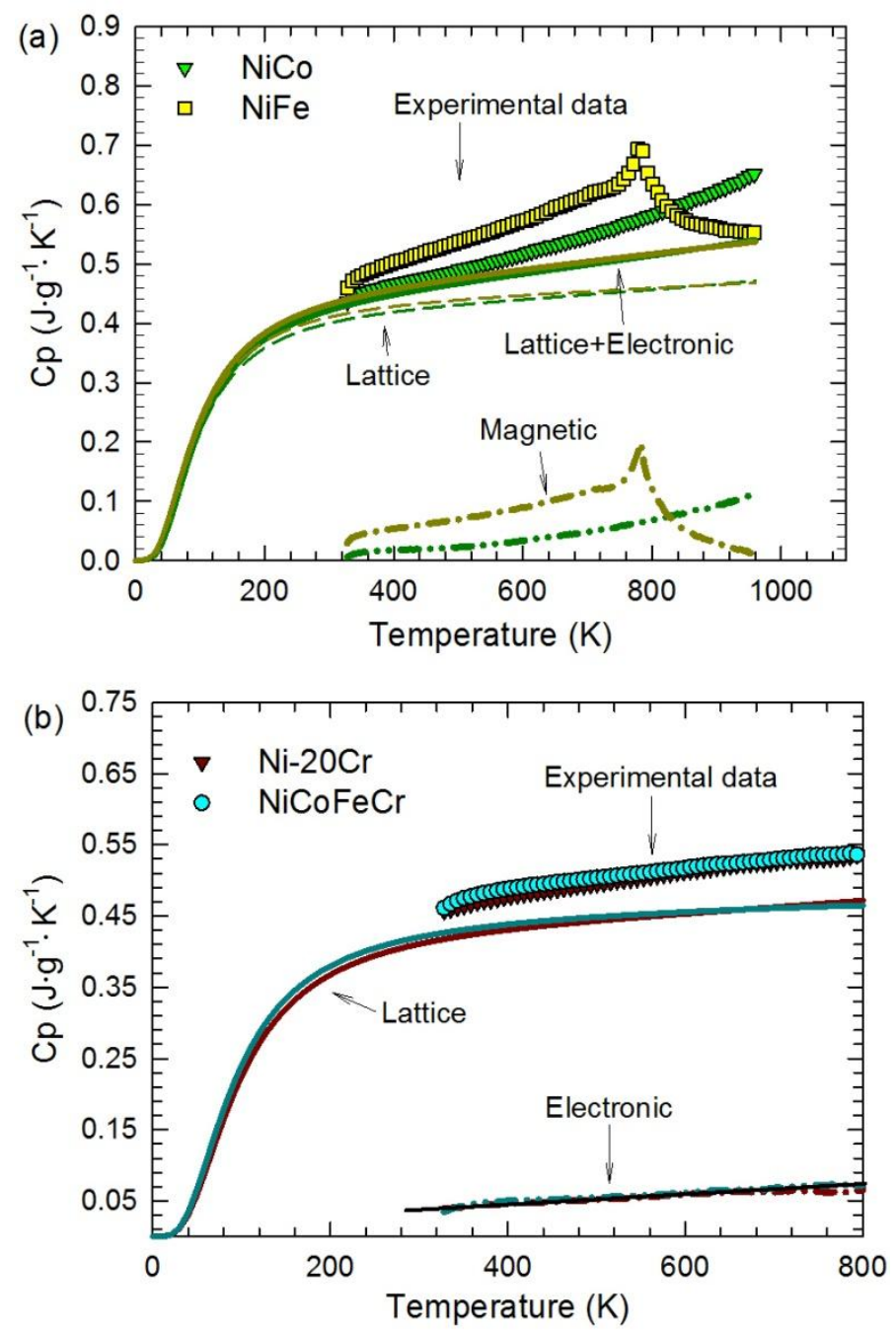

Fig. 4. The measured and calculated (lattice) specific heat of (a) $\mathrm{NiCo}$ and $\mathrm{NiFe}$, and (b) $\mathrm{Ni}-20 \mathrm{Cr}$ and $\mathrm{NiCoFeCr}$. The electronic and magnetic contributions are also separated for each alloy. 

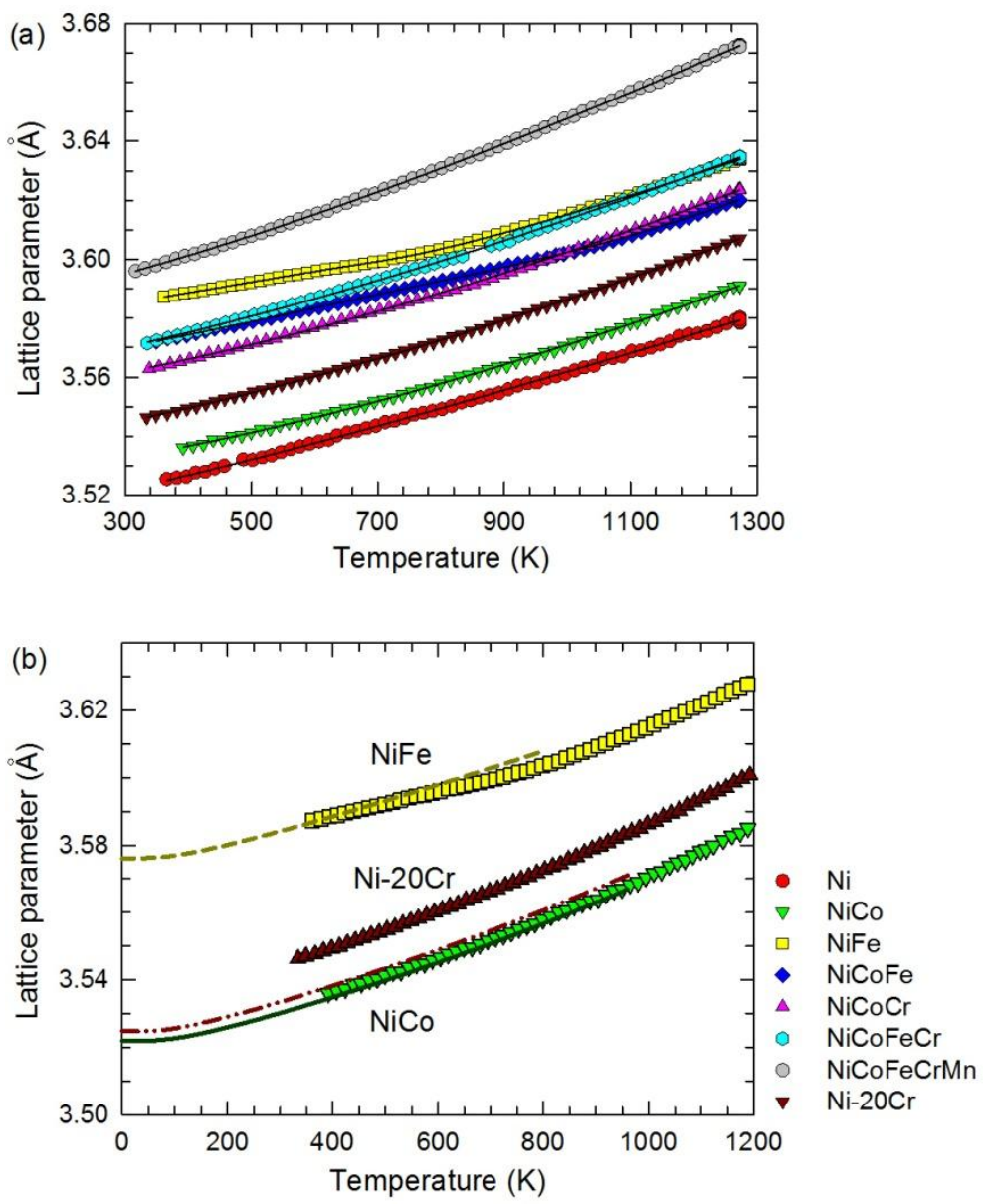

Fig. 5. (a) Neutron diffraction measured lattice parameter as a function of temperature of $\mathrm{Ni}$ and the alloys. (b) Comparison between the calculated lattice parameter (lines) and the experimental data (symbols) of $\mathrm{NiCo}, \mathrm{NiFe}$, and $\mathrm{Ni}-20 \mathrm{Cr}$. The legends apply for both figures. 


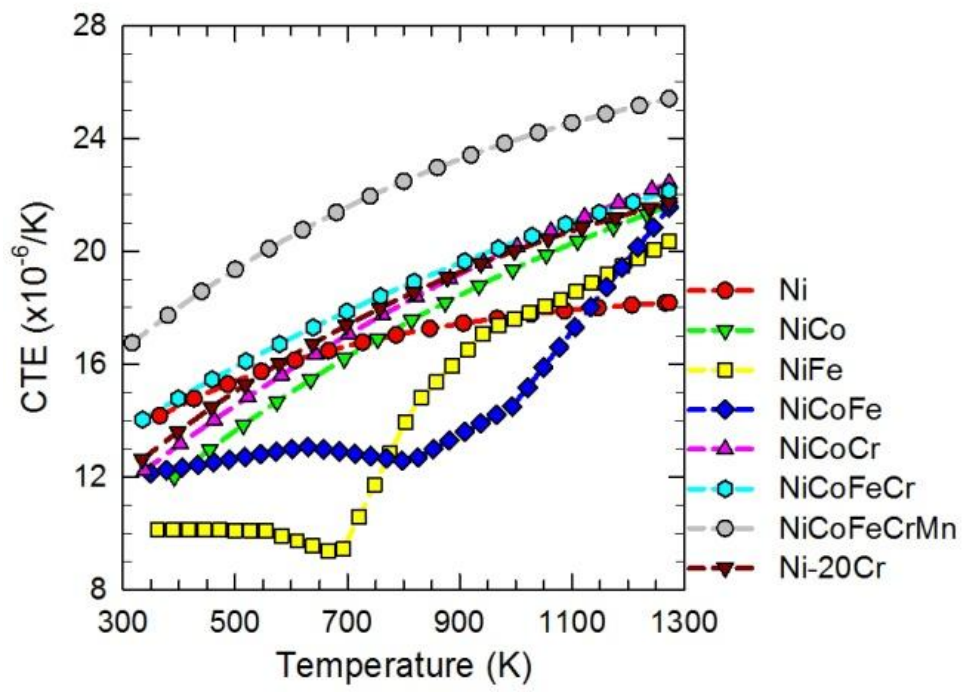

Fig. 6. The coefficient of thermal expansion of the alloys derived from the data shown in Fig. 5a. 

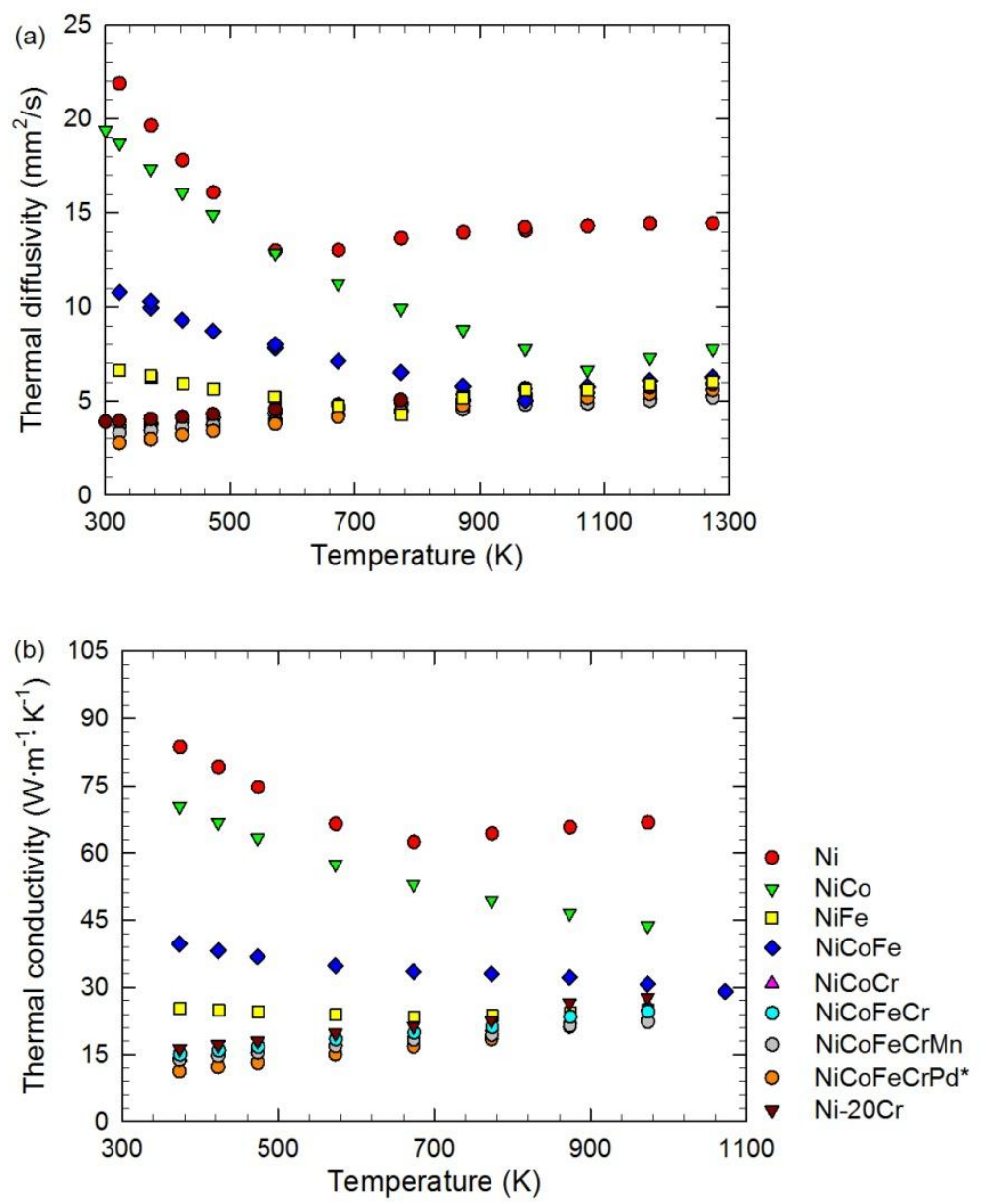

Fig. 7. (a) Thermal diffusivity and (b) the calculated thermal conductivity of $\mathrm{Ni}$ and $\mathrm{Ni}$-containing single phase concentrated solid solution alloys. *Room temperature density of $\mathrm{NiCoFeCrPd}$ was used since the temperature dependent data are not available, which may lead to as much as $3 \%$ error in the high temperature region. Legends apply to both figures. 


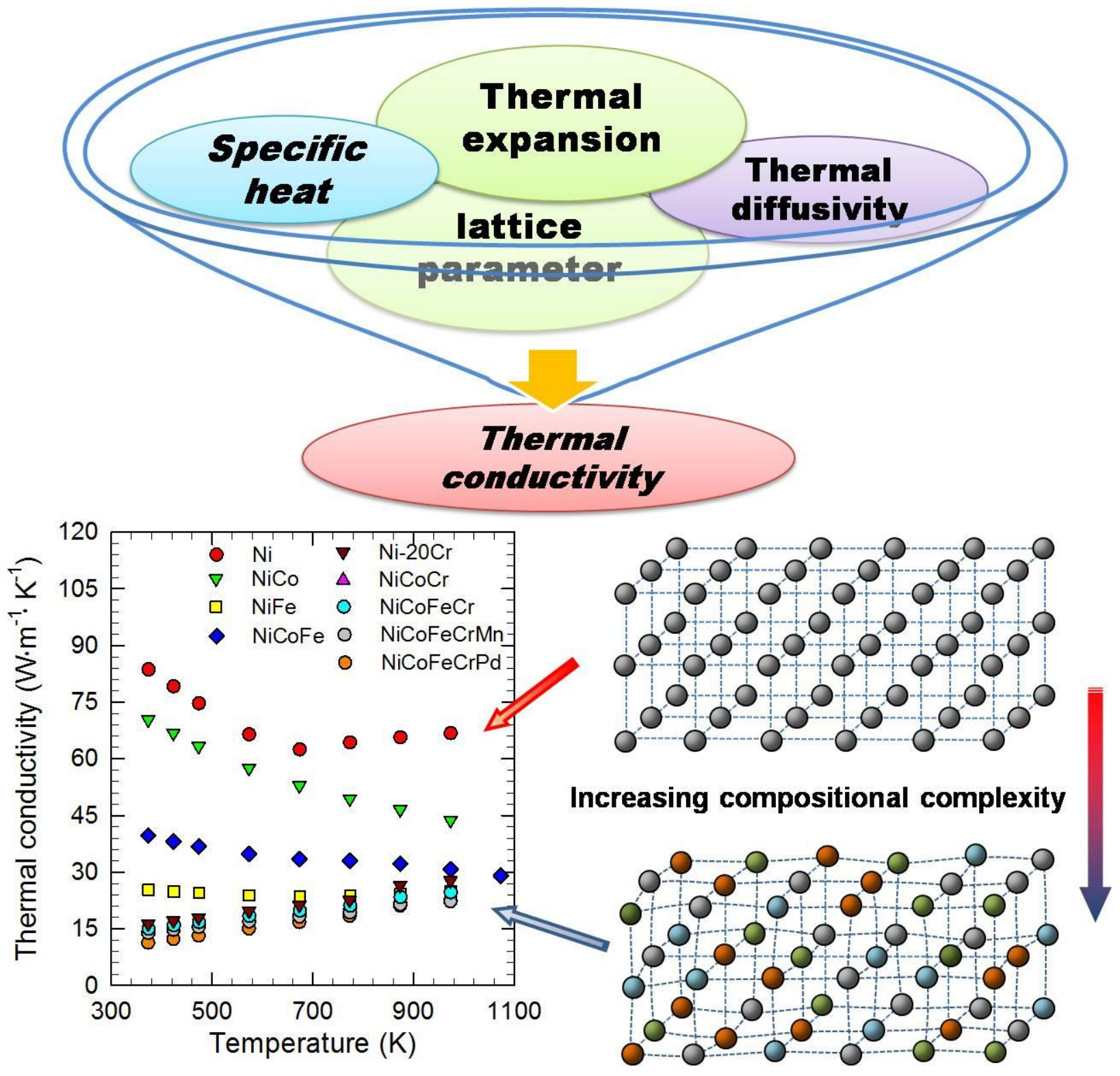

